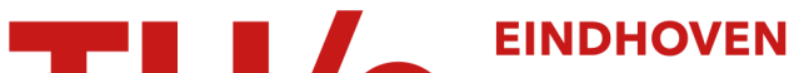 \\ UNIVERSITY OF \\ TECHNOLOGY
}

\section{Dynamic light scattering in shear flows}

Citation for published version (APA):

Hoppenbrouwers, M. B., \& Water, van de, W. (1998). Dynamic light scattering in shear flows. Physics of Fluids, 10(9), 2128-2136. https://doi.org/10.1063/1.869734

DOI:

10.1063/1.869734

Document status and date:

Published: 01/01/1998

\section{Document Version:}

Publisher's PDF, also known as Version of Record (includes final page, issue and volume numbers)

\section{Please check the document version of this publication:}

- A submitted manuscript is the version of the article upon submission and before peer-review. There can be important differences between the submitted version and the official published version of record. People interested in the research are advised to contact the author for the final version of the publication, or visit the $\mathrm{DOI}$ to the publisher's website.

- The final author version and the galley proof are versions of the publication after peer review.

- The final published version features the final layout of the paper including the volume, issue and page numbers.

Link to publication

\section{General rights}

Copyright and moral rights for the publications made accessible in the public portal are retained by the authors and/or other copyright owners and it is a condition of accessing publications that users recognise and abide by the legal requirements associated with these rights.

- Users may download and print one copy of any publication from the public portal for the purpose of private study or research.

- You may not further distribute the material or use it for any profit-making activity or commercial gain

- You may freely distribute the URL identifying the publication in the public portal.

If the publication is distributed under the terms of Article $25 \mathrm{fa}$ of the Dutch Copyright Act, indicated by the "Taverne" license above, please follow below link for the End User Agreement:

www.tue.nl/taverne

\section{Take down policy}

If you believe that this document breaches copyright please contact us at:

openaccess@tue.nl

providing details and we will investigate your claim. 


\title{
ARTICLES
}

\section{Dynamic light scattering in shear flow}

\author{
Marc Hoppenbrouwers and Willem van de Water \\ Department of Physics, Eindhoven University of Technology, P.O. Box 513, 5600 MB, Eindhoven, \\ the Netherlands
}

(Received 13 January 1998; accepted 7 May 1998)

\begin{abstract}
We describe a new dynamic light scattering technique for measuring diffusion in sheared suspensions. It involves a scattering geometry with two crossing laser beams. A detailed analysis of the correlation function of scattered light is given. The viability of our method is demonstrated in an experiment where the effect of Taylor diffusion on the scattered light correlation function is measured. (C) 1998 American Institute of Physics. [S1070-6631(98)00409-7]
\end{abstract}

\section{INTRODUCTION}

Dynamic light scattering is a powerful tool for probing fluctuations in dispersions. Information from such experiments comes in the form of the dynamic structure factor which is a central quantity for characterizing these fluctuations. On the other hand, light scattering is also a fine tool for measuring the velocity field in flowing media. The tantalizing question is if light scattering methods can be devised that can quantify both the mean flow and the diffusive motion in flowing dispersions. Such a method would be of key relevance to probe the coupling of concentration fluctuations to an external shear field in heterogeneous media. This is a problem of great current interest as shear may alter macromolecular conformations, may induce structural changes (melting, crystallization) in dense colloidal suspensions ${ }^{1}$ and may even induce diffusion in dispersions where thermal agitation plays no role. ${ }^{2}$

The principle of dynamic light scattering is that random changes in the relative position of particles, for example, due to Brownian motion, result in a fluctuating scattered light intensity. These fluctuations are characterized by measurement of the scattered light time-correlation function. A wellknown practical application is the measurement of particle properties through measurement of the diffusion coefficient. Accessible time scales follow from the requirement that the change of relative position be comparable to the wavelength of light. In a sheared suspension another source of fluctuations is the random passage of particles through the observation volume.

The difficulty of light scattering techniques to probe diffusion in shear flow may be appreciated by comparing the magnitude of the time scales involved. Assuming that no mean flow is present, the scattered light time scale $\tau_{F}$ of the random passage fluctuations is set by the linear size $d$ of the scattering volume as $\tau_{F}=\lambda /(d \gamma)$, with $\lambda$ the wavelength of light and $\gamma$ the shear rate. In order to observe diffusion, its own time scale must be much more rapid than $\tau_{F}$. The influence of shear on diffusion is felt on time scales $\tau \approx \gamma^{-1}$, however, $\tau / \tau_{F}=d / \lambda \gg 1$. In this case, measured correlation functions are overwhelmed by the flow time-scale $\tau_{F}$, and a simple light scattering setup is not suited for measuring diffusion in shear flow.

In this paper we will discuss a light scattering method that overcomes this difficulty and gives access to diffusion in a flowing fluid with a velocity gradient. As a model system we will study a solution of Brownian spheres which undergoes a precisely defined shear in a Couette device. Because this model system can be analyzed in detail, we will be able to asses the precision of our experiment and, in general, the feasibility of our experimental method.

Brownian motion in shear flow was analyzed many years ago by Taylor $^{3}$ who studied the spreading of soluble substances in a fluid that flows slowly through narrow tubes. In this case, spreading is the consequence of both molecular diffusion in the radial direction combined with the (Poiseuille) flow profile in the tubes. It was verified both experimentally and theoretically that the axial spreading of the cross-sectionally averaged concentration followed an ordinary diffusion equation with an effective diffusion coefficient which is a function of the velocity profile and the molecular diffusion constant.

This is surprising in view of the simple argument that predicts the diffusive spreading in a linear shear to be anomalous. Imagine a shear flow in the $x$ direction with a velocity gradient in the $y$ direction. A random Brownian displacement in the $y$ direction couples to the shear field and results in a mean squared displacement $\left\langle x^{2}\right\rangle \sim t^{h}$, where the exponent $h$ has the anomalous value $h=3 / 2$, which reflects the combined action of random walk $\left(\left\langle y^{2}\right\rangle \sim t\right)$ and deterministic flight $\left\langle x^{2}\right\rangle \sim t^{2}$. Taylor's result is special in that a radial average of the concentration is considered in tubes which are so narrow that radial diffusion is swift compared to convection by the mean flow.

In general, therefore, the diffusion of Brownian particles in shear flow is anomalous: $\left\langle x^{2}\right\rangle \sim t^{3}$. It is a remarkable coincidence that the same anomalous exponent holds for the 
spreading of particles in a turbulent flow, where it is known as Richardson's law. ${ }^{4}$ The derivation of this law involves dimensional arguments related to the cascade picture of fully developed turbulence.

An early attempt to measure diffusion in shear flow using dynamic light scattering was reported Ackerson and Clark, who verified the fundamental problem sketched above. ${ }^{5}$ Experiments at very small shear rates, where $\tau_{F} \gg \tau$, and where diffusion remains virtually unaffected by the flow field, were reported in Ref. 6.

The sensitivity of dynamic light scattering to shear can be used to devise techniques for the measurement of shear in a point. This is an attractive alternative to computing it from the difference between flow velocities measured in two spatially separated points. This technique was pioneered by Fuller et al. ${ }^{7}$ and applied to optically turbid flows by $\mathrm{Wu}$ et $a .^{8}$ An interesting variant involving a cross-correlation technique was demonstrated by Keveloh and Staude. ${ }^{9}$ Our work was inspired by their paper which already suggested a way to measure Brownian motion. In fact, our usage of two beams is complementary to the cross-correlation technique described in Ref. 9, but it has the advantage of a much better definition of the scattering volume. In Sec. V we will show that precise control of the location of the scattering volume is a key ingredient of the success of our method. A related application of dynamic light scattering is the measurement of shear fluctuations in turbulence. ${ }^{10}$ In this case, the correlation function reflects the statistical properties of turbulence. However, as will be explained in Sec. II, in a standard singlebeam setup these properties are convoluted with the poorly known geometry of the scattering volume.

In the next section we present a detailed analysis of the scattered-light correlation function of diffusing particles in a shear flow. Correlation functions are the product of a diffusive factor and a structure factor which has a nontrivial geometry-dependent time dependence. On basis of this analysis we describe in Sec. III the practical realization of a setup in which both diffusion and shear can be measured. Our test case consists of a dispersion of Brownian particles in shear flow. The predictions for the correlation function for this case are summarized in Sec. IV. The experiment is described in Sec. V and results are discussed in Sec. VI. A central result is that our method is indeed suited for quantifying diffusion in a shear flow. A challenge that could be met only partly is the accurate determination of Taylor diffusion; our results are only in qualitative agreement with the predictions.

\section{LIGHT SCATTERING}

Imagine a shear flow with suspended Brownian particles. A laser beam is focussed in the flow and the scattered light with wave vector $\mathbf{k}$ is detected. We will analyze the temporal fluctuations of the scattered light intensity in terms of the intensity correlation function. The time dependence of the correlation function is determined both by particle diffusion and particle convection due to shear. In a conventional light scattering setup, where a single light beam and a single detector is used, and thus a single scattered wave vector is involved, the convective contribution completely obscures that of diffusion. We will demonstrate that the associated time scales can be unravelled instead in a setup using two scattered wave vectors $\mathbf{k}_{1}$ and $\mathbf{k}_{2}$.

In this case, the scattered electric field is the sum of fields corresponding to the two scattered wave vectors

$$
E(t)=E\left(\mathbf{k}_{1}, t\right)+E\left(\mathbf{k}_{2}, t\right),
$$

where the fields are the sum of contributions of $N$ individual particles

$$
E\left(\mathbf{k}_{m}, t\right)=\sum_{j=1}^{N} E_{\mathbf{k}_{m}}\left(\mathbf{r}_{j}(t)\right) e^{i \mathbf{k}_{m} \cdot \mathbf{r}_{j}(t)},
$$

with $\mathbf{r}_{j}(t)$ the position of sphere $j$ at time $t$. The amplitude factor $E_{\mathbf{k}_{m}}(\mathbf{r})$ describes the distribution of the electric field amplitude over the scattering volume. It is determined by the intensity profile of both laser beams and the geometry of the scattered light collection optics. It follows from Eq. (1) that the scattered electric field correlation function is the sum of four contributions

$$
\left\langle E^{*}(0) E(t)\right\rangle=g_{11}(t)+g_{12}(t)+g_{21}(t)+g_{22}(t),
$$

with

$$
g_{12}(t)=\sum_{i, j}^{N}\left\langle E_{\mathbf{k}_{1}}^{*}\left(\mathbf{r}_{i}(0)\right) E_{\mathbf{k}_{2}}\left(\mathbf{r}_{j}(t)\right) e^{-i \mathbf{k}_{1} \cdot \mathbf{r}_{i}(0)+i \mathbf{k}_{2} \cdot \mathbf{r}_{j}(t)}\right\rangle
$$

and similarly for the other terms in Eq. (3). The brackets \langle\rangle in Eq. (4) denote ensemble averaging. We assume that particles are independent so that the sum in Eq. (4) has only contributions with $i=j$.

The correlation function in Eq. (4) embodies two sources of fluctuation: one involves the fluctuations of the phase factor, and the other one the time dependence of the amplitude factor. We will now argue that the phases fluctuate much more rapidly than the amplitudes, so that amplitude fluctuations can be ignored in most cases. Diffusive motion with diffusion constant $D$ induces fluctuations of the phase with time constant $\tau_{D}=\lambda^{2} / D$, where $\lambda$ is the wavelength of light. In contrast, the fluctuations of the amplitude factor are on a time scale $d^{2} / D$, where $d$ is the linear size of the scattering volume. Because $\lambda \ll d$, diffusive amplitude fluctuations can be ignored with respect to the corresponding phase fluctuations.

The flow of particles through the scattering volume $V$ induces an amplitude time scale $\tau_{A}$. In case that a mean flow is present, $\tau_{A}=d / U_{V}$, where $U_{V}$ is the mean flow velocity in the scattering volume. The effect of mean flow can be eliminated by selecting $U_{V}=0$ in the experiment. In that case $\tau_{A}$ is determined by the velocity gradient, $\tau_{A}=\gamma^{-1}$. Again, the corresponding time scales of the phase fluctuations are a factor of $\lambda / d$ shorter. Accordingly, we approximate the correlation function by

$$
g_{12}(t)=\sum_{i}^{N}\left\langle E_{\mathbf{k}_{1}}^{*}\left(\mathbf{r}_{i}(0)\right) E_{\mathbf{k}_{2}}\left(\mathbf{r}_{i}(0)\right) e^{-i \mathbf{k}_{1} \cdot \mathbf{r}_{i}(0)+i \mathbf{k}_{2} \cdot \mathbf{r}_{i}(t)}\right\rangle,
$$

and similarly for the other contributions to $\left\langle E^{*}(0) E(t)\right\rangle$. 
The coupling between diffusion and shear is gauged by the product $\gamma t$. If $t$ is taken to be the diffusion time scale $\tau_{D}$, it follows that the coupling is significant if $\gamma \tau_{D}=\tau_{D} / \tau_{A}$ is of order unity. (The ratio $\tau_{D} / \tau_{A}$ is commonly called the Peclet number.) As $\tau_{A}$ is a time scale of amplitude fluctuations, it now seems that our desire to ignore amplitude fluctuations is incompatible with our interest in the coupling between diffusion and shear. However, it will appear that in our experiment we measure the correlation function at constant $\gamma t$. Below, we will explicate the effect of convection on the amplitude factor and demonstrate that it is a function of $\gamma t$ only. Therefore, we expect that the effect of number fluctuations, that is the random passage of particles through the scattering volume, is a constant, time-independent background in our experiment.

The phase fluctuations in Eq. (5) still contain a deterministic part that is due to the convection of particles through the scattering volume. We therefore write

$$
\mathbf{r}_{j}(t)=\widetilde{\mathbf{r}}_{j}(t)+\int_{0}^{t} \mathbf{u}\left(\mathbf{r}_{j}(0), t^{\prime}\right) d t^{\prime},
$$

where $\mathbf{u}(\mathbf{r}, t)$ is the flow field and $\tilde{\mathbf{r}}_{j}(t)$ is the fluctuating position of particle $j$ with its velocity taken relative to the flow velocity at $\mathbf{r}_{j}(0)$. We assume a stationary linear flow field with velocity gradient $\boldsymbol{\Gamma}$

$$
\mathbf{u}(\mathbf{r})=\mathbf{u}_{0}+\boldsymbol{\Gamma} \cdot \mathbf{r} .
$$

The correlation function $g_{12}$ then becomes

$$
\begin{aligned}
g_{12}(t)= & e^{i \mathbf{k}_{2} \cdot \mathbf{u}_{0} t} \sum_{j}\left\langle E_{\mathbf{k}_{1}}^{*}\left(\mathbf{r}_{j}(0)\right) E_{\mathbf{k}_{2}}\left(\mathbf{r}_{j}(0)\right)\right. \\
& \left.\times e^{-i\left(\Delta \mathbf{k}-t \mathbf{k}_{2} \cdot \Gamma\right) \cdot \mathbf{r}_{j}(0)} e^{-i \mathbf{k}_{2} \cdot\left[\mathbf{r}_{j}(0)-\tilde{\mathbf{r}}_{j}(t)\right]}\right\rangle,
\end{aligned}
$$

where $\Delta \mathbf{k}=\mathbf{k}_{1}-\mathbf{k}_{2}$.

The average in Eq. (8) is the product of a factor due to (deterministic) particle convection and a diffusive factor. The first factor can be written as an integral over the scattering volume

$$
S_{12}(\tilde{\mathbf{k}})=\int_{V} E_{\mathbf{k}_{1}}^{*}(\mathbf{r}) E_{\mathbf{k}_{2}}(\mathbf{r}) e^{-i \tilde{\mathbf{k}} \cdot \mathbf{r}} d \mathbf{r},
$$

with $\tilde{\mathbf{k}}=\Delta \mathbf{k}-t \mathbf{k}_{2} \cdot \boldsymbol{\Gamma}$. The structure factor $S_{12}$ (and thus $g_{12}$ ) is only nonzero when the scattering volumes corresponding to $\mathbf{k}_{1}$ and $\mathbf{k}_{2}$ actually overlap. Further, in the case of an infinitely extended and flat scattering volume the structure factor reduces to a delta function

$$
S_{12}(\tilde{\mathbf{k}}) \propto \delta(\tilde{\mathbf{k}}), \quad \text { or } \quad \Delta \mathbf{k}=t \mathbf{k}_{2} \cdot \boldsymbol{\Gamma} .
$$

In this case the correlation function $g_{12}(t)$ has a delta peak at a time delay $t^{*}$ given by $\Delta \mathbf{k}-t^{*} \mathbf{k}_{2} \cdot \boldsymbol{\Gamma}=0$, where the alignment of $\Delta \mathbf{k}$ and $\mathbf{k}_{2} \cdot \boldsymbol{\Gamma}$ is of obvious relevance. For a scattering volume of finite extent $d$, the peak in $g_{12}(t)$ at $t=t^{*}$ has a width $\Delta t \approx \lambda / d \gamma$.

The diffusive factor is the correlation

$$
F\left(\mathbf{k}_{2}, t\right) \equiv\left\langle e^{-i \mathbf{k}_{2} \cdot[\mathbf{r}(0)-\widetilde{\mathbf{r}}(t)]}\right\rangle .
$$

It is the Fourier-transformed conditional probability of finding a sphere at position $\mathbf{r}(t)$ at time $t^{\prime}=t$ under the condition that it was at $\mathbf{r}(0)$ at time $t^{\prime}=0$. The height of the peak in $g_{12}(t)$ is the overlap integral $\int E_{\mathbf{k}_{1}}^{*}(\mathbf{r}) E_{\mathbf{k}_{2}}(\mathbf{r}) d \mathbf{r}$ times the value of the diffusive factor taken in $t=t^{*}$.

So far, we have been concerned with the electric field correlation functions $g_{i j}(t)$. In an actual experiment, however, we measure light intensities:

$$
I(t)=\left[E^{*}\left(\mathbf{k}_{1}, t\right)+E^{*}\left(\mathbf{k}_{2}, t\right)\right]\left[E\left(\mathbf{k}_{1}, t\right)+E\left(\mathbf{k}_{2}, t\right)\right],
$$

with the associated intensity correlation function $G(t)$ $=\langle I(0) I(t)\rangle$. In the case of Gaussian fluctuations, the following relation holds:

$$
G(t)=1+\left|g_{11}(t)+g_{12}(t)+g_{21}(t)+g_{22}(t)\right|^{2} .
$$

Equation (13) follows when the size $d$ of the scattering volume is much larger than the wavelength $\lambda$ of light and when particles are assumed independent. In case of an infinitely extended scattering volume, the partial correlations $g_{i j}(t)$ peak at different time delays, and the right-hand side of Eq. (13) simplifies considerably.

Several salient contributions can be recognized in Eq. (13):

I. The term

$$
\begin{aligned}
g_{11}(t) g_{22}^{*}(t)= & e^{i\left(\mathbf{k}_{1}-\mathbf{k}_{2}\right) \cdot \mathbf{u}_{0} t} S_{11}\left(\mathbf{k}_{1} \cdot \boldsymbol{\Gamma} t\right) \\
& \times S_{22}^{*}\left(\mathbf{k}_{2} \cdot \boldsymbol{\Gamma} t\right) F\left(\mathbf{k}_{1}, t\right) F^{*}\left(\mathbf{k}_{2}, t\right)
\end{aligned}
$$

forms, together with its complex conjugate, the well-known description of laser Doppler velocimetry (LDV). The correlation function oscillates as $\cos \left[\left(\mathbf{k}_{1}-\mathbf{k}_{2}\right) \cdot \mathbf{u}_{0} t\right]$ with an envelope that is determined both by particle diffusion and particle convection in the shear field. Of course, LDV is only practical in cases where $u_{0} \neq 0$, in which case the envelope is determined by particle transit through the scattering volume.

II. In a single-beam setup, the effect of LDV is absent in a homodyne measurement of the correlation function, and

$$
G(t)=1+|S(\mathbf{k} \cdot \Gamma t) F(\mathbf{k}, t)|^{2} .
$$

As was argued in Sec. I, the decay of the structure factor $S(\mathbf{k} \cdot \boldsymbol{\Gamma} t)$ is in general much faster than that of the correlation function $F(\mathbf{k}, t)$, so that the effect of Brownian motion is almost always completely obscured. A Herculean effort to unfold $F(\mathbf{k}, t)$ in single-beam measurements has been reported by Ackerson and Clark. ${ }^{5}$ Indeed, when $S(\mathbf{k})$ is known precisely, one could try to unravel $F(\mathbf{k}, t)$ from the measured correlation function. However, precise knowledge about $S(\mathbf{k})$ in an experiment is extremely difficult, as it depends on the light collector geometry. It does therefore not surprise that Ackerson and Clark $^{5}$ were unable to detect Brownian motion in shear flow.

III. In a two-beam setup, the appropriate contribution to the correlation function $G(t)$ is

$$
g_{12}^{*}(t) g_{12}(t)=\left|S_{12}\left(\Delta \mathbf{k}-\mathbf{k}_{2} \cdot \boldsymbol{\Gamma} t\right) F\left(\mathbf{k}_{2}, t\right)\right|^{2} .
$$

The structure factor $S_{12}$ determines the observation time $t^{*}$ as

$$
t^{*}=\frac{\Delta \mathbf{k} \cdot\left(\mathbf{k}_{2} \cdot \boldsymbol{\Gamma}\right)}{\left\|\mathbf{k}_{2} \cdot \boldsymbol{\Gamma}\right\|^{2}} \equiv \frac{\Delta k_{\|}}{\left\|\mathbf{k}_{2} \cdot \boldsymbol{\Gamma}\right\|},
$$




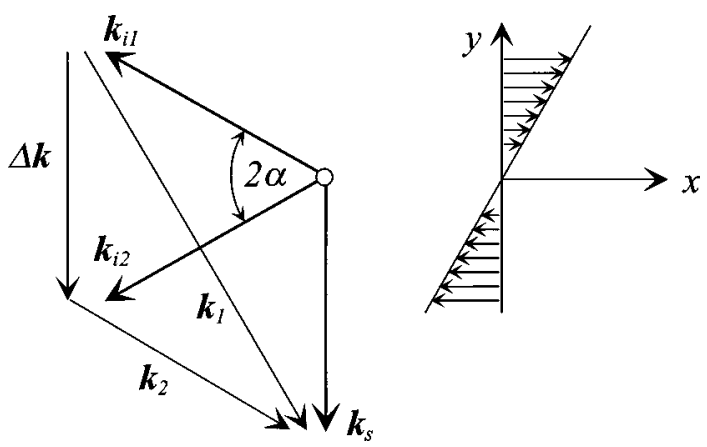

FIG. 1. Schematic view of the light scattering setup. Two laser beams with wave vectors $\mathbf{k}_{i_{1}}$ and $\mathbf{k}_{i_{2}}$ cross under an angle $2 \alpha$ in the scattering volume. Scattered light with wave vector $\mathbf{k}_{s}$ is observed. Accordingly, the scattering wave vectors are $\mathbf{k}_{1}$ and $\mathbf{k}_{2}$, respectively. Shear is arranged in the $y$ direction.

which can be tuned by changing the difference scattering vector $\Delta \mathbf{k}$ and keeping the shear rate $\gamma$ fixed, or by changing $\gamma$ at fixed $\Delta \mathbf{k}$. In the latter case, also the product $\gamma t^{*}$ is fixed. The time $t=t^{*}$ at which the diffusion $F\left(\mathbf{k}_{2}, t\right)$ is probed, now depends on $\gamma$ and the function $F\left(\mathbf{k}_{2}, t\right)$ itself may change if there exists a coupling between diffusion and shear.

It must be emphasized that for our method to work optimally, the vector $\Delta \mathbf{k}$ must be parallel to $\mathbf{k}_{2} \cdot \boldsymbol{\Gamma}$. Misalignment results in signal reduction which for the case of a Gaussian scattering volume with width $d$ would be proportional to $\exp \left[-\left(\Delta k_{\perp} d\right)^{2} / 4\right]$ where $\Delta k_{\perp}$ is the component of $\Delta \mathbf{k}$ perpendicular to the vector $\mathbf{k}_{2} \cdot \boldsymbol{\Gamma}$.

IV. If the two scattering volumes corresponding to the scattering wave vectors $\mathbf{k}_{1}$ and $\mathbf{k}_{2}$ do not overlap, the timedelayed contribution $g_{12}^{*}(t) g_{12}(t)$ [Eq. (16)] vanishes and we are left with the laser-Doppler contribution

$$
\begin{aligned}
g_{11}(t) g_{22}^{*}(t)= & e^{i\left(\mathbf{k}_{1} \cdot \mathbf{u}_{1}-\mathbf{k}_{2} \cdot \mathbf{u}_{2}\right) t} S_{11}\left(\mathbf{k}_{1} \cdot \mathbf{\Gamma} t\right) \\
& \times S_{22}^{*}\left(\mathbf{k}_{2} \cdot \boldsymbol{\Gamma} t\right) F\left(\mathbf{k}_{1}, t\right) F^{*}\left(\mathbf{k}_{2}, t\right),
\end{aligned}
$$

where $\mathbf{u}_{1}$ is the mean flow velocity in scattering volume 1 , and similarly for $\mathbf{u}_{2}$. Because $\mathbf{u}_{1} \neq \mathbf{u}_{2}$, nonoverlapping scattering volumes result in an oscillatory correlation function. Due to the structure factor $S_{k m}$ these oscillations can only be observed near zero time delay, $t \leqslant \lambda / d \gamma$. The occurrence of oscillations, therefore, is an indication of poor alignment.

\section{PRACTICAL REALIZATION}

The geometry of our experiment is sketched in Fig. 1. Shear is in the $y$ direction only with a single nonzero $\Gamma_{x y}$ $=\gamma$ component of the velocity gradient. Two incident beams with wave vectors $\mathbf{k}_{i_{1}}$ and $\mathbf{k}_{i_{2}}$ cross under an angle $2 \alpha$ and we observe the scattered light in a direction perpendicular to $\mathbf{k}_{i_{1}}+\mathbf{k}_{i_{2}}$. From Eq. (17) it follows that we probe the correlation function $F\left(\mathbf{k}_{1}, t\right)$ at $t=t^{*}$, where $t^{*}$ is given by ${ }^{11}$

$$
t^{*} \mathrm{~s}(2 \tan \alpha) / \gamma \text {. }
$$

Scanning the correlation function $F\left(\mathbf{k}_{1}, t\right)$ is possible by scanning $t^{*}$. As explained, this can be done either by changing $\alpha$ or by changing $\gamma=\Gamma_{x y}$. However, varying the beam crossing angle $\alpha$ will affect the shape of the scattering volume and thus the structure factor $S_{k m}$. It is very difficult to apply the appropriate geometric corrections to $S_{k m}$ in an actual experiment. Therefore, scanning $t^{*}$ is realized by scanning the shear rate $\gamma$. As in our experiment the beam crossing angle could not be made very large, reaching short time scales was limited by the occurrence of hydrodynamic instabilities at large $\gamma$. The influence of shear on diffusion is gauged by the value of the product $\gamma t^{*}=2 \tan \alpha$, which, in principle, can be made arbitrarily large, but was quite small in our experiment.

The width $\Delta t$ of the cross-correlation peak at $t^{*}$ is

$$
\Delta t=t^{*} \frac{\lambda}{2 d \tan \alpha} .
$$

Longer delay times, therefore, demand a better statistical accuracy.

In summary, our method allows us to scan time at a constant value of $\gamma t$. An advantage is its insensitivity to number fluctuations which would give rise to a timedependent amplitude correlation in Eq. (4). In the case of shear without mean flow, it is possible to derive an expression for the contribution to the correlation function due to number fluctuations

$$
G_{N}(t)=\frac{1}{n d^{2}\left[1+\frac{1}{4}(\gamma t)^{2}\right]^{1 / 2}},
$$

where $n$ is the particle density and where a Gaussian scattering volume with width $d$ has been assumed. As expected, $G_{N}(t)$ depends on $\gamma t$ only and merely gives a constant background in our method. Moreover, the effect of number fluctuations is only felt at very low particle concentrations. Here and earlier we have assumed a Gaussian beam profile. As the scattering volume is also defined by pinholes, the actual intensity profile is more complicated. Results as Eq. (21), therefore, have only illustrative value.

The principle of our light scattering technique follows from a rather elaborate analysis of the scattered light correlation function. It is dissatisfying that we have found no simple heuristic principle for this experimental technique. An (incomplete) analogy can be made with the often used picture of ordinary laser-Doppler velocimetry. This explains the observed oscillatory Doppler intensity signal by imagining particles that traverse the fringes of an interference pattern of two crossing laser beams. Similarly, the presence of a velocity gradient would result in a white spectrum of Doppler frequencies, the Fourier transform of which gives a delta peak at zero time delay in the correlation function. However, to appreciate that this delta peak is actually at a time delay $t^{*} \mathrm{~s}(2 \tan \alpha) / \gamma$, a complete analysis of the correlation function is needed. In Ref. 9 an attempt at a heuristic explanation is made using the concept of "speckle path." The problem, however, is that such an explanation needs mastering another concept, which for us did not come intuitively. 


\section{BROWNIAN MOTION IN SHEAR FLOW}

Our model system for studying the coupling of fluctuations to external shear is a solution of Brownian particles subjected to simple shear

$$
\boldsymbol{\Gamma}=\left(\begin{array}{lll}
0 & \gamma & 0 \\
0 & 0 & 0 \\
0 & 0 & 0
\end{array}\right) \text {. }
$$

In absence of shear these particles diffuse with $D$ $=k_{B} T /(6 \pi \eta a)$, where $a$ is the particle radius, $\eta$ the viscosity, $T$ the temperature, and $k_{B}$ the Boltzmann constant. For this particular form of $\Gamma$, the correlation function $F(\mathbf{k}, t)$ is $^{7,12}$

$$
F(\mathbf{k}, t)=e^{-D\left[k_{x}^{2}\left(1+\frac{1}{3}(\gamma t)^{2}\right)-\gamma t k_{x} k_{y}+k_{y}^{2}+k_{z}^{2}\right] t},
$$

from which it readily follows

$$
\begin{gathered}
\left\langle x^{2}\right\rangle=2 D t\left[1+(\gamma t)^{2} / 3\right], \quad\langle x y\rangle=D \gamma t^{2}, \\
\left\langle y^{2}\right\rangle=2 D t, \text { and }\left\langle z^{2}\right\rangle=2 D t,
\end{gathered}
$$

which shows the anomalous diffusion in the flow direction.

So far we have ignored the effect of hydrodynamic retardation, that is that the flow field around a sphere needs a time of the order of $\tau_{\mathrm{VAC}}$ (the velocity autocorrelation time) to adjust to its motion. This time scale is very short, $\tau_{\mathrm{VAC}}$ $=(6 \pi \eta a / M)^{-1}=\mathscr{Q}\left(10^{-9} \mathrm{~s}\right)$, where $M$ is the mass of a Brownian particle. In general, allowance of hydrodynamic retardation severely complicates the theory of Brownian motion. It is remarkable, therefore, that for Brownian motion in shear flow, exact solutions that embody retardation effects can be reached. ${ }^{13}$ These authors found a serious modification of the long-time behavior of $\left\langle x^{2}(t)\right\rangle$ :

$$
\begin{aligned}
\left\langle x^{2}(t)\right\rangle= & 2 D t\left\{1+\frac{(\gamma t)^{2}}{3}\left[1-\frac{18}{5}\left(\frac{2}{\pi} \frac{\rho}{\rho_{p}}\right)\left(\frac{t}{\tau_{\mathrm{VAC}}}\right)^{-1 / 2}\right.\right. \\
& \left.\left.+\left(\frac{33}{4} \frac{\rho}{\rho_{p}}-6\right)\left(\frac{t}{\tau_{\mathrm{VAC}}}\right)^{-1}+\mathcal{Q}\left(t^{-3 / 2}\right)\right]\right\},
\end{aligned}
$$

where $\rho / \rho_{p}$ is the ratio of fluid and particle density. However, as for our experiments $\tau_{\mathrm{VAC}}=\mathscr{C}\left(10^{-9}\right) \mathrm{s}$, and accessible time delays are $\mathscr{Q}\left(10^{-4}\right) \mathrm{s}$, experimental observation of these retardation effects is elusive. In order to observe these effects it is necessary to detect motion on very small time scales. The difficulty is that then the sphere displacements and accordingly the scattered light signal are prohibitively small. In recent years, the technique of diffusing wave spectroscopy has been proposed to access these short times. ${ }^{8}$

\section{EXPERIMENTS}

Figure 2 shows our experimental setup. Shear flow is generated in a Couette device with two counter-rotating glass cylinders that are driven by separate stepper motors. This allows precise control of the rotation rate of each cylinder. The radius $R_{o}$ of the outer cylinder was $R_{o}=25.2 \mathrm{~mm}$, and that of the inner cylinder $R_{i}=20.0 \mathrm{~mm}$. The azimuthal flow field $u(r)$ of a Couette flow is

$$
u(r)=A r-B / r,
$$

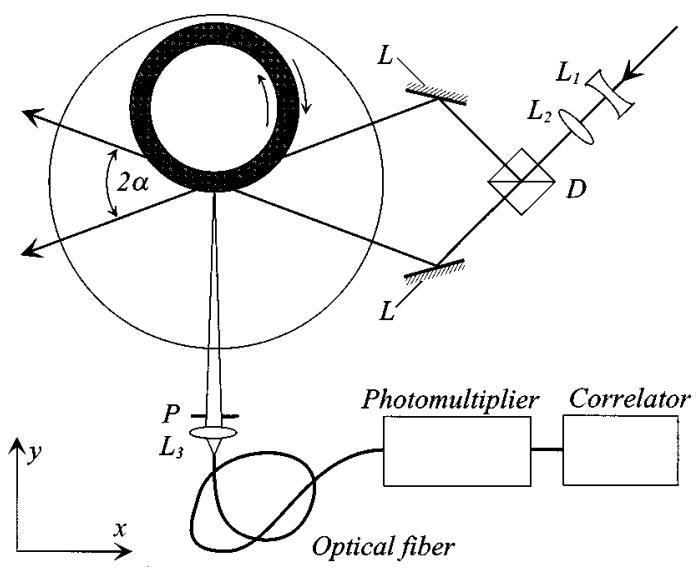

FIG. 2. Schematic view (not to scale) of the experimental setup. The Couette shear flow (shaded) is contained between two counter rotating cylinders. A laser beam is split into two (coherent) halves in $D$. The two beams are focussed in the scattering volume using the lenses $L_{1}$ and $L_{2}$ where they cross under an angle $2 \alpha$. The crossing angle can be varied by sliding the mirrors over $L$. Scattered light is observed through pinhole $P$ and imaged by lens $L_{3}$ onto a $0.1 \mathrm{~mm}$ diameter optical fiber that acts as a second pinhole. A large glass cylinder containing an index matched fluid is placed concentrically with the scattering volume.

with $A=\left(R_{o}^{2} \Omega_{o}-R_{i}^{2} \Omega_{i}\right) /\left(R_{o}^{2}-R_{i}^{2}\right) \quad$ and $\quad B=R_{o}^{2} R_{i}^{2}\left(\Omega_{o}\right.$ $\left.-\Omega_{i}\right) /\left(R_{o}^{2}-R_{i}^{2}\right)$, where $\Omega_{o}$ and $\Omega_{i}$ are the rotation rates of the outer and inner cylinder, respectively. In the scattering volume we approximate the flow by simple shear $u(y)=u_{0}$ $+\gamma y$ with $\gamma=\left(\Omega_{o} R_{o}-\Omega_{i} R_{i}\right) /\left(R_{o}-R_{i}\right)$.

As light sources we have used a HeNe-laser operating at $\lambda=632.8 \mathrm{~nm}$ or a frequency-stabilized $\mathrm{Ar}^{+}$-ion laser at $\lambda$ $=514.5 \mathrm{~nm}$. The primary laser beam is split into equal fractions by a nonpolarizing beamsplitter. A negative (focal length $f=-40 \mathrm{~mm})$ and a positive lens $(f=100 \mathrm{~mm})$ are used to focus both secondary beams at the location of the scattering volume. The distance between beam splitter and scattering volume is fixed. This way, we may vary the angle between the secondary beams by extending or reducing the length of both legs $L$. The beams are easily aligned and focused by placing a pinhole (diameter $0.01 \mathrm{~mm}$ ) at the projected position of the scattering volume. The scattering volume is located exactly midway between the two counterrotating cylinders.

The scattered light collection optics consists of a pinhole (diameter $1.0 \mathrm{~mm})$ and a lens $(f=100 \mathrm{~mm})$ which images the scattering volume onto the end of a multimode optical fiber (100 $\mu \mathrm{m}$ core diameter) that acts as a second pinhole. The length $d_{x}$ of the scattering volume is computed to be 0.2 $\mathrm{mm}$. The diameter $\left(d_{y} \approx 30 \mu \mathrm{m}\right)$ is determined by the width of the beam focus. Scattered light is detected with help of a photo multiplier whose output photo pulses are fed to a realtime digital correlator.

To prevent refraction of the incoming laser beams and the scattered light, a cylindrical container is placed around the two rotating cylinders such that its center coincides with the scattering volume. Both this container and the inner rotating cylinder are filled with a mixture of dibutylphtalate (88.6\%) and hexanol (11.4\%) whose index of refraction $n$ $=1.4820$ matches that of the used glass. 


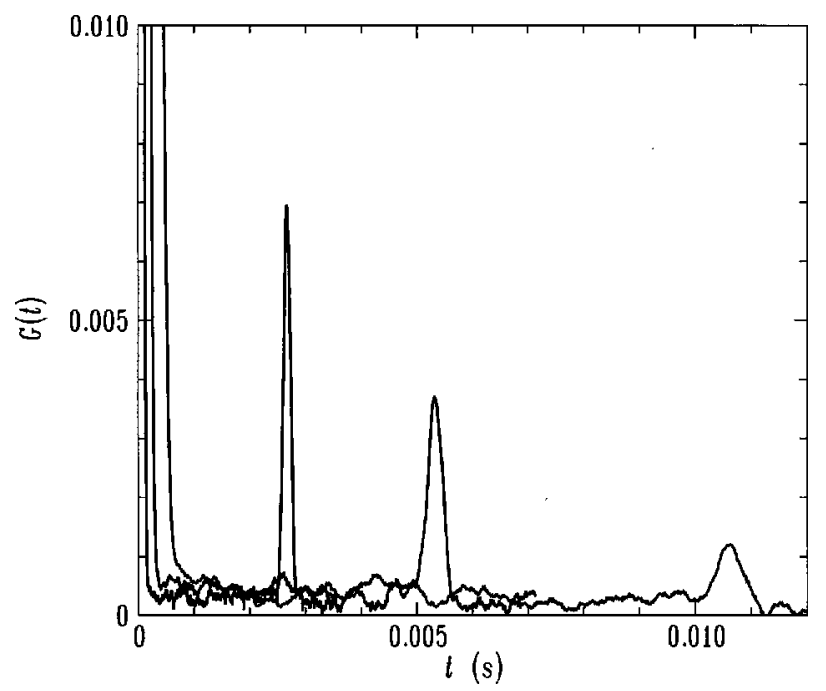

FIG. 3. Series of three autocorrelation functions measured at three shear rates, $\gamma=54.3 \mathrm{~s}^{-1}, \gamma=27.14 \mathrm{~s}^{-1}$, and $\gamma=13.6 \mathrm{~s}^{-1}$ for the curves that have peaks at $t^{*}=2.66 \times 10^{-3} \mathrm{~s}, t^{*}=5.32 \times 10^{-3} \mathrm{~s}$, and $t^{*}=1.06 \times 10^{-4} \mathrm{~s}$, respectively. The beam crossing angle is $\alpha=4.13^{\circ}$. The baseline [ $G(t=\infty)$ $\approx 1$ ] has been subtracted from $G(t)$.

The overall time scale of the experiment is proportional to the solvent viscosity. The shortest reachable time scale, however, is determined by the onset of secondary flow at large $\gamma$. Long times can be reached with slow shear rates, but then the cross-correlation peak at $t^{*}$ is broad and the stability and statistical accuracy becomes a problem. We have therefore found it preferable to work at low viscosities that correspond to short diffusion time scales. The price paid, of course, is earlier onset of secondary flow at the shortest time scale. For the solvent, we selected a mixture whose index of refraction matched that of the glass cylinders, whose density approximately equaled that of the dispersed particles such as to prevent sedimentation, and whose viscosity was small. These requirements could be met with a mixture of $\gamma$ picoline $(83.7 \%)$ and water $(16.3 \%)$. We used a dispersion of $200 \mathrm{~nm}$ diameter polystyrene spheres in the water-picoline mixture. Single scattering conditions were realized at the used particle concentration $c=3.7 \times 10^{-4}$.

Figure 3 shows a series of measured autocorrelation functions at varying shear rates. The correlation functions have a peak at zero time delay, and a cross-correlation peak at $t^{*}=(2 \tan \alpha) / \gamma$. The width of both peaks is seen to decrease with increasing shear rate.

The correlation function shown in Fig. 3 is the result of a careful collector alignment and a careful adjustment of the flow field in the Couette device. The collector was placed at a fixed $y$ distance from the scattering volume, but its $x$ position could be varied such as to align the collector line of sight exactly with the point where the two laser beams cross. As explained in Sec. II, gross misalignment results in an oscillatory correlation function near $t=0$. First-order alignment is aimed at eliminating these oscillations. The $x$ position is then fine tuned by maximizing the height of the secondary peak.

If the average flow velocity is nonzero in the scattering volume, the peak is obscured by noise due to the flow of

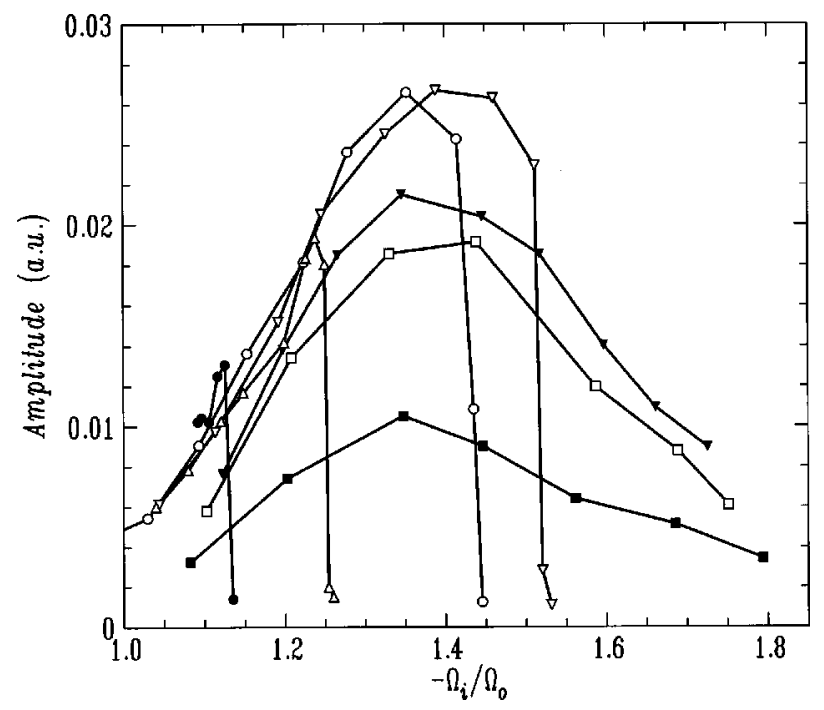

FIG. 4. Amplitude of the cross-correlation peak as a function of the frequency ratio of inner and outer cylinders $\Omega_{i} / \Omega_{o}$ at various values of $\Omega_{o}$, $\Omega_{o}=2.00 \mathrm{rad} \mathrm{s}^{-1}(\mathbf{\square}), \Omega_{o}=3.99 \mathrm{rad} \mathrm{s}^{-1}(\square), \Omega_{o}=4.70 \mathrm{rad} \mathrm{s}^{-1}(\boldsymbol{\nabla}), \Omega_{o}$ $=7.87 \mathrm{rad} \mathrm{s}^{-1}(\bigcirc), \Omega_{o}=7.12 \mathrm{rad} \mathrm{s}^{-1}(\nabla), \Omega_{o}=9.66 \mathrm{rad} \mathrm{s}^{-1}(\triangle), \Omega_{o}$ $=11.50 \mathrm{rad} \mathrm{s}^{-1}(\bullet)$. The maximum shear rate increases with increasing $\Omega_{o}$. The rapid drop off at large shear rates signifies the onset of secondary flow in the Couette device.

particles through the scattering volume. It is therefore important to position the scattering volume at the radius where $u_{0}=0$. For a typical shear rate of $\gamma=40 \mathrm{~s}^{-1}$, a misalignment of $250 \mu \mathrm{m}$ corresponds to a mean velocity of $u_{0}$ $=10^{-2} \mathrm{~ms}^{-1}$. The associated convection time scale $\tau_{A 1}$ $\approx 10 \mathrm{~ms}$ would then be of the order of the diffusion time scale, and our light scattering technique would not work.

When changing the shear rate, the point where $u_{0}=0$ can be kept in place by adjusting the angular frequencies of both inner and outer cylinder such that the ratio of frequencies remains constant $\left(\Omega_{i} / \Omega_{o}=-1.41\right.$ for our cylinders). Figure 4 shows the amplitude of the cross-correlation peak as a function of the ratio of inner and outer rotation rates $\Omega_{i} / \Omega_{o}$ at various fixed values of $\Omega_{o}$. It is seen that the maximum peak amplitude indeed corresponds to $\Omega_{i} / \Omega_{o}$ $=-1.41$ and therefore to the situation where there is no mean flow in the scattering volume. The value of $\Omega_{i} / \Omega_{o}$ $=-1.41$ corresponds to larger shear $\gamma$ for larger $\Omega_{o}$, and thus to shorter time delays. Consequently, the height of the maximum of the curves increases for increasing $\Omega_{o}$. The sharp cutoff in the curves is due to the onset of the Taylor instability.

\section{RESULTS}

First, we will demonstrate the viability of our light scattering method: it can be used to quantify diffusive motion in the presence of shear. A practical application would be the measurement of the radius of particles which are suspended in a shear flow. We will show that this can be done with a precision of approximately $10 \%$. Next, we will demonstrate experimental evidence for the coupling between diffusion and shear. This is a challenge because the product $\gamma t$ in our experiments is small due to geometric constraints. In our 
TABLE I. Summary of experimental results shown in Figs. 5 and 6 . The figure indication points to the frames of Fig. 6. The directly determined beam crossing angle $\alpha_{d}$ can be compared to the value $\alpha_{m}$ that was determined from the shift of the cross-correlation peak with applied shear (see Fig. 5). The particle diameter $d_{m}$ that was found from the measured correlation functions (Fig. 6) should be compared to the known value of 0.204 $\mu \mathrm{m}$. The measured shear-induced decay rate $\Gamma_{s_{m}}$ must be compared to the value $\Gamma_{s}$ that is known from Taylor dispersion theory. In all cases we used light from a HeNe laser at $632.8 \mathrm{~nm}$, except for case (b) where light of 514.5 nm was used.

\begin{tabular}{ccccccc}
\hline \hline Fig. 6 & $\gamma \tau$ & $\alpha_{d}\left({ }^{\circ}\right)$ & $\alpha_{m}\left({ }^{\circ}\right)$ & $d_{m}(\mu \mathrm{m})$ & $\Gamma_{s_{m}}\left(\mathrm{~s}^{-1}\right)$ & $\Gamma_{s}\left(\mathrm{~s}^{-1}\right)$ \\
\hline $\mathrm{a}$ & 0.123 & 3.58 & 3.52 & 0.191 & $28 \pm 10$ & 12.2 \\
$\mathrm{~b}$ & 0.148 & 4.13 & 4.23 & 0.194 & $13 \pm 10$ & 18.5 \\
$\mathrm{c}$ & 0.172 & 4.92 & 4.92 & 0.168 & $15 \pm 10$ & 17.2 \\
$\mathrm{~d}$ & 0.282 & 8.02 & 8.03 & 0.196 & $28 \pm 10$ & 29.1 \\
\hline \hline
\end{tabular}

setup, the smallest delay time that can be reached is limited by the onset of flow instabilities. We will demonstrate experimentally that this actually occurs at the known boundary of the Taylor instability. The various experimental parameters and results are listed in Table I.

Four measurements of the time delay $t^{*}$, height and width of the cross-correlation peak as a function of the shear rate $\gamma$ were done at four different beam crossing angles $\alpha$ and therefore at four different values of $\gamma t^{*}$. Before each measurement, the viscosity of the solution was checked by measuring the time autocorrelation function of the scattered light of the two probing beams in the absence of Couette flow and taking the diameter of the spheres as a known value.

The time delay $t^{*}$ of the cross-correlation peak depends linearly on the inverse shear rate $1 / \gamma$ as $t^{*}=(2 \tan \alpha) / \gamma$. Figure 5 demonstrates that this relation is accurately satisfied in our experiment. From the slope of the straight line which was determined in a least-squares procedure, the beam crossing angle $\alpha$ could be computed. As Table I demonstrates, it

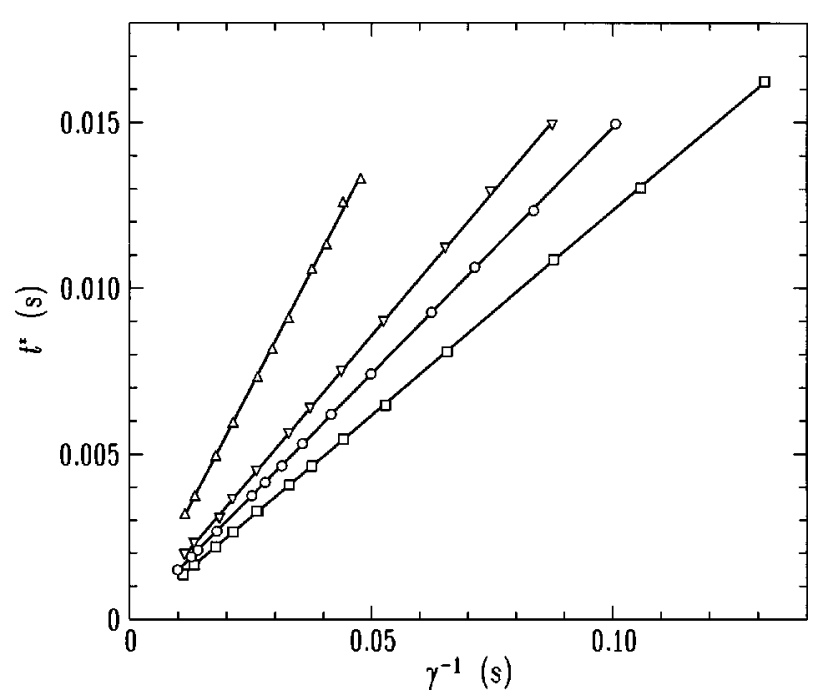

FIG. 5. The position $t^{*}$ of the cross-correlation peak $\tau_{0}$ as a function of the inverse applied shear rate $1 / \gamma$ at various beam crossing angles. The symbols correspond to $\alpha=3.52^{\circ}(\square), \alpha=4.23^{\circ}(\bigcirc), \alpha=4.92^{\circ}(\nabla), \alpha=8.03^{\circ}(\triangle)$. The lines are least squares fits, whose slope $s$ is used to determine the beam crossing angle as $\alpha=\tan ^{-1}(s / 2)$.
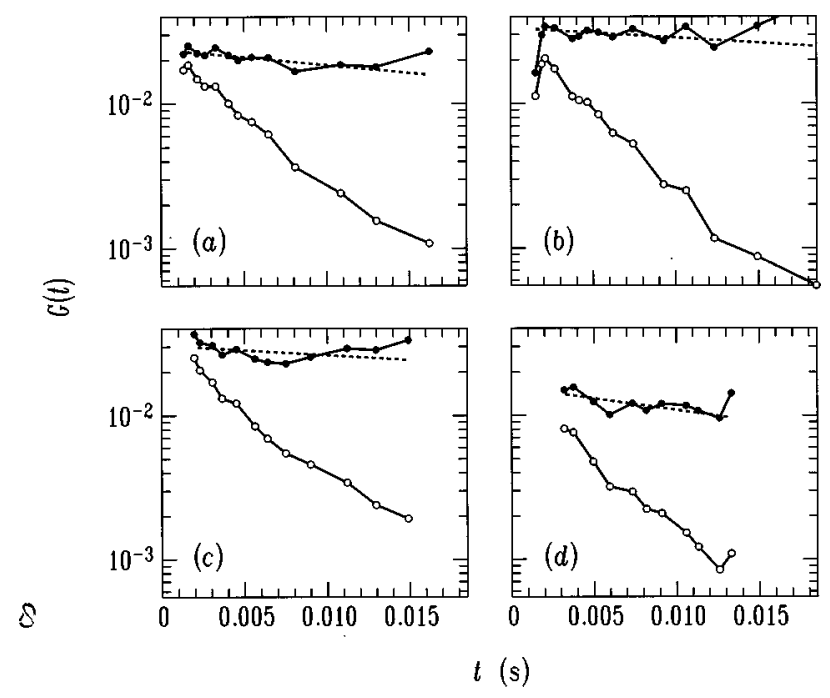

FIG. 6. Open circles: the height of the cross-correlation peak in the (normalized) correlation function $G(t)$ above the baseline as a function of the time delay. Closed dots: $G(t) \times e^{-D k^{2} t}$. The dashed lines are determined in a least squares procedure. The beam crossing angle $\alpha$ is (a) $3.58^{\circ}$, (b) $4.23^{\circ}$, (c) $4.92^{\circ}$, and (d) $8.03^{\circ}$.

agrees to within $1 \%$ with the directly measured value. These results again demonstrate that this light scattering method provides a very accurate measurement of the shear in a single point. $^{9}$

The height of the cross-correlation peak as a function of its position $t^{*}$ traces the diffusive correlation function $F\left(\mathbf{k}, t^{*}\right)$. This is demonstrated in Fig. 6 which shows that the height decays approximately exponentially with delay time. These correlation functions can be compared to correlation functions that are measured directly in the absence of shear. However, the indirect measurement causes much larger statistical fluctuations. The deviations from exponentiality at large time delays are most probably due to noise in measured correlation functions.

The correlation functions were used to determine the particle diameter by fitting Eq. (23) with the particle diameter as an unknown. As is seen, the measured particle diameters agree to within $15 \%$ with the known value.

A very interesting question is if we can experimentally observe the influence of shear on diffusion. In fact, this is already implicit in the determination of the sphere diameter, but here we like to address this question more directly. It follows from Eq. (23) that the correlation function is

$G(t) \sim e^{-D k_{2}^{2} t} e^{-\Gamma_{s} t}$ with $\Gamma_{s}=D\left[k_{2_{x}}^{2}(\gamma t)^{2} / 3-k_{2_{x}} k_{2_{y}}(\gamma t)\right]$.

The shear-induced decay rate $\Gamma_{s}$ is a fraction of order $\gamma t$ of the ordinary diffusive decay; this is a small number $(\gamma t$ $=\mathscr{Q}(0.1))$ in our experiments. In Fig. 6 we have also plotted $e^{D k_{2}^{2} t} \times G(t)$ using the known value of the particle diameter for computing the diffusion coefficient $D$. From Eq. (27) it follows that the remainder should be exponentially decreasing as $e^{-\Gamma_{s} t}$. In almost all cases the remainder is found to decrease with a decay rate $\Gamma_{s_{m}}$ which is comparable to the predicted one. Because the remainder $e^{D k_{2}^{2} t} \times G(t)$ is found 


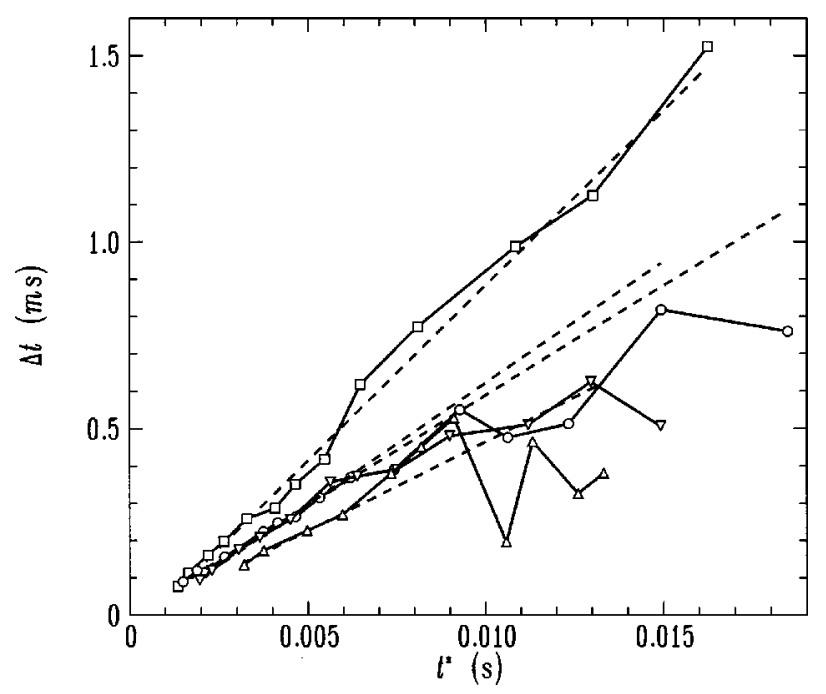

FIG. 7. Width of the secondary peak as a function of its time delay $t^{*}$. The symbols correspond to beam crossing angles $\alpha=3.52^{\circ}(\square), \alpha=4.23^{\circ}(\bigcirc)$, $\alpha=4.92^{\circ}(\nabla), \alpha=8.03^{\circ}(\triangle)$. The dashed lines are linear least-squares fits whose slope $s$ is used to determine the beam width as $d_{y}=\lambda /(2 s \tan \alpha)$. The computed widths are $d_{y}=55 \mu \mathrm{m}$ for $\alpha=3.52^{\circ}, d_{y}=59 \mu \mathrm{m}$ for $\alpha=4.23^{\circ}$, $d_{y}=56 \mu \mathrm{m}$ for $\alpha=4.92^{\circ}$, and $d_{y}=47 \mu \mathrm{m}$ for $\alpha=8.03^{\circ}$.

to deviate from exponential at short and long times, the estimated error in the measured $\Gamma_{s_{m}}$ is large. We conclude that the influence of shear on diffusion is just barely visible in our experiments.

The width of the cross-correlation peak increases with increasing delay time (decreasing shear rate) according to $\Delta t=t^{*} \lambda /(2 d \tan \alpha)$. In Fig. 7 we show the measured widths $\Delta t\left(t^{*}\right)$. From the observed linear dependence at small time delays we can infer a width $d_{y}$ of the scattering volume which varies from $d_{y}=47 \mu \mathrm{m}$ to $d_{y}=59 \mu \mathrm{m}$. These values are comparable to the computed width of the focussed laser beams. At long time delays, large fluctuations in the measured $\Delta t$ are evident, especially at the largest beam crossing angle. It reflects the decreasing signal to noise ratio at increasing beam crossing angles. Measurement of the peak height appears to be less sensitive to this problem.

In our experiment, time delays are scanned through variation of the shear rate $\gamma$. In a Couette device, secondary (nonazimuthal) flow emerges when a critical value of $\gamma$ is exceeded. Of relevance is the Taylor number that is defined as

$$
T=\frac{4 \Omega_{i}^{2} d^{4}}{\nu^{2}} \frac{\left(\rho_{r}^{2}-\mu^{2}\right)}{1-\rho_{r}^{2}},
$$

with $\rho_{r}=R_{i} / R_{o}, \mu=\Omega_{i} / \Omega_{o}$ and $d=R_{o}-R_{i}$. In our case where the ratios $\rho_{r}$ and $\mu$ are fixed, increasing $\gamma$ necessarily leads to exceeding the critical value of the Taylor number. This is not an important restriction of our light scattering method because $T$ strongly depends on the gap width and it is not difficult to construct a Couette device with a much narrower gap.

The stability diagram of simple Couette flow is sketched in Fig. 8 in terms of $A R_{i}^{2} / B$ and $B / \nu$ (the constants $A$ and $B$ have been introduced in Sec. V). In our experiments, crossing of the stability boundary causes sudden collapse of the

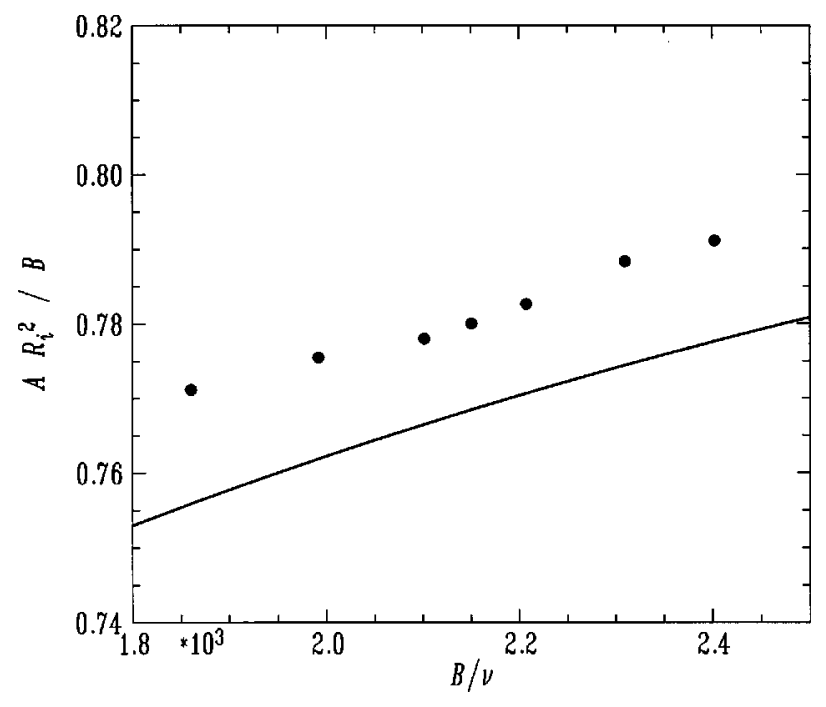

FIG. 8. Above the full line Couette flow is unstable. The experimental dots signify the collapse of the cross-correlation peak.

cross-correlation peak. An example of this collapse was already shown in Fig. 4. The locus of this collapse was determined in an experiment in which the rotation frequency of the inner cylinder was determined at which the crosscorrelation peak suddenly vanished, while the rotation frequency of the outer cylinder was kept constant. The points in Fig. 8 lie just above the stability boundary, which means that our flow becomes unstable earlier than predicted by theory. This may be explained by the imperfections of the setup, especially by the fact that our cylinders were slightly nonconcentric.

\section{CONCLUSIONS}

We have demonstrated the feasibility of a light scattering method that can quantify diffusion in shear flow. Our method is based on the unfolding of time scales associated with diffusion and shear using a heterodyne technique. In this way, the outcome of the measurement is not longer dominated by the geometric shape of the scattering volume. This dependence has thwarted earlier attempts to use dynamic light scattering for the measurement of diffusive motion in shear.

Our technique enabled us to measure the diffusion coefficient with reasonable accuracy. Taylor diffusion is the modification of thermal diffusion by shear. In our experiments we were just able to observe its effect on measured correlation functions. The strength of the effect is gauged by the product $\gamma t$, whose value was limited by the maximum attainable beam crossing angle. Larger values resulted in unacceptable signal loss. Clearly, a redesign of the optics is needed to cure this problem. Otherwise, there is no bound on the value of $\gamma t$.

The range of accessible time delays is determined by the largest shear rate before secondary flow sets in. In our experiments this occurred relatively early because of the wide gap between the cylinders of our Couette apparatus. However, by diminishing it (much) shorter times can easily be reached. 


\section{ACKNOWLEDGMENTS}

We thank Jan Niessen for technical assistance. We gratefully acknowledge financial support by the "Nederlandse Organisatie voor Wetenschappelijk Onderzoek (NWO)', and "Stichting Fundamenteel Onderzoek der Materie (FOM)."

${ }^{1}$ L. B. Chen, C. F. Zukoski, B. J. Ackerson, H. J. M. Hanley, G. C. Straty, J. Barker, and C. J. Glinka, "Structural changes and orientational order in a sheared colloidal suspension," Phys. Rev. Lett. 69, 688 (1992); J. Liu, D. A. Weitz, and B. J. Ackerson, "Coherent crystallography of shearaligned crystals of hard-sphere colloids,"' Phys. Rev. E 48, 1106 (1993). ${ }^{2}$ See, for example, D. T. Leighton and A. Acrivos, "Measurement of shearinduced tracer diffusivity in concentrated suspensions of spheres," J. Fluid Mech. 177, 109 (1987); S. I. Madanshetty, A. Nadim, and H. A. Stone, "Experimental measurement of shear-induced diffusion in suspensions using long time data," Phys. Fluids 8, 2011 (1996).

${ }^{3}$ G. I. Taylor, "Dispersion of soluble matter in solvent flowing slowly through a tube," Proc. R. Soc. London, Ser. A 219, 186 (1953).

${ }^{4}$ A recent discussion of Richardson's law can be found in U. Frisch, Turbulence (Cambridge University, Cambridge, 1995), p. 102.
${ }^{5}$ B. J. Ackerson and N. A. Clark, "Dynamic light scattering at low rates of shear,'” J. Phys. (Paris) 42, 929 (1981).

${ }^{6} \mathrm{Y}$. Tsunashima, "Particle diffusion and convection observed by dynamic light scattering from aqueous suspensions of polystyrene-latex particles in simple shear flow,'” J. Phys. Soc. Jpn. 61, 2763 (1992).

${ }^{7}$ G. G. Fuller, J. M. Rallison, R. L. Schmidt, and L. G. Leal, “The measurement of velocity gradients in laminar flow by homodyne lightscattering spectroscopy," J. Fluid Mech. 100, 555 (1980).

${ }^{8}$ X-L. Wu, D. J. Pine, P. M. Chaikin, J. S. Huang, and D. A. Weitz, "Diffusing-wave spectroscopy in a shear flow,"' J. Opt. Soc. Am. B 7, 15 (1990).

${ }^{9}$ C. Keveloh and W. Staude, "Determination of velocity gradients with scattered light cross-correlation measurements," Appl. Opt. 22, 333 (1983).

${ }^{10} \mathrm{P}$. Tong and W. I. Goldburg, "Relative velocity fluctuations in turbulent flows at moderate Reynolds numbers. I. Experimental," Phys. Fluids 31, 2841 (1988).

${ }^{11}$ In our setup $\Delta \mathbf{k}$ points in the negative $y$ direction, $g_{21}$ instead of $g_{12}$ pertains and we probe $F\left(\mathbf{k}_{1}, t\right)$ instead of $F\left(\mathbf{k}_{2}, t\right)$.

${ }^{12}$ R. T. Foister and T. G. M. van der Ven, "Diffusion of Brownian particles in shear flows," J. Fluid Mech. 96, 105 (1980).

${ }^{13}$ P. P. J. M. Schram and S. A. Trigger, "Brownian motion in shear flows, harmonic potentials and colloidal crystals," Physica B 228, 91 (1996). 